\title{
Maximum lifetime body mass index is the appropriate predictor of knee and hip osteoarthritis
}

\author{
Sabine Patricia Singer ${ }^{1}$ Dietmar Dammerer ${ }^{2} \cdot$ Martin Krismer $^{2}$. \\ Michael C. Liebensteiner ${ }^{2}$
}

Received: 2 August 2017 / Published online: 27 October 2017

(C) The Author(s) 2017. This article is an open access publication

\begin{abstract}
Introduction In light of inconsistencies in the literature, this study aimed to investigate the relationship between obesity (current and historic) and osteoarthritis (OA) of the knee or hip.

Materials and methods We examined 99 people (knee OA, hip OA and controls), age $>50$ years, in a case-control study. The current weight, height and waist circumference were measured on site, and detailed weight changes over their lifetime were based on questionnaires and standardized interviews. We used binomial logistic regression to determine the predictive value for an osteoarthritis group membership of each derived indicator.

Results An increase in 'maximum-BMI' increased the odds ratio for both knee OA (OR 1.2; CI 1.1-1.4; $p=0.005$; $R^{2}=0.36$ ) and hip OA (OR 1.2; CI 1.0-1.3; $p=0.027$; $R^{2}=0.16$ ). Current BMI was significantly associated with knee OA but not with hip OA. A high "minimum-BMI" (over the age of 18 years) had the highest odds ratio of all calculated indicators for both osteoarthritis groups.
\end{abstract}

Dietmar Dammerer

dietmar.dammerer@tirol-kliniken.at

Sabine Patricia Singer

kontakt@dr-singer.net

Martin Krismer

martin.krismer@tirol-kliniken.at

Michael C. Liebensteiner

michael.liebensteiner@i-med.ac.at

1 Private Practice for Orthopaedics, Am Isarkanal 24/6/7, 81379 Munich, Germany

2 Department of Orthopaedic Surgery, Medical University of Innsbruck, Anichstr. 35, 6020 Innsbruck, Austria
Conclusions Based on our findings, it is concluded that the maximum BMI over one's lifespan is a better predictor of $\mathrm{OA}$ of the hip or the knee than the current BMI. The knee joint seems to be more sensitive to obesity as current BMI was associated only with knee OA but not with hip OA.

Keywords Obesity $\cdot$ Body mass index $\cdot$ Knee osteoarthritis · Hip osteoarthritis · Overweight

\section{Introduction}

Osteoarthritis (OA) of the knee and the hip are common conditions in the community, characterized by the degeneration of cartilage and its underlying bone with a high impact on the health-related quality of life [1]. As incidence and prevalence of OA rise with advancing age [2], extended life expectancy will result in greater numbers of $\mathrm{OA}$ increasing the financial burden on the health care systems considerably $[3,4]$.

$\mathrm{OA}$ is a complex multifactorial disease [5, 6]. Among the predominant risk factors that contribute to OA are age and weight [6-8]. Several studies examined the association of the body mass index (BMI) as well as other indicators of overweight and obesity (e.g. waist-circumference) with OA of knee and hip [9-12]. While several studies confirmed a significant association between BMI and knee OA but not for hip OA [11, 12], others found associations between BMI and hip OA $[9,10]$.

Due to the above-mentioned inconsistencies in the literature, it was the aim of the study to further investigate the relationship between obesity (current and historic) and OA of the knee and hip. It was hypothesized that there would be a significant association between the 'maximum-BMIover-one's-lifespan' ('maximum-BMI') and OA of the knee 
or hip (Hypothesis 1). It was further hypothesized that also the 'current BMI' would be linked to OA of the knee or hip (Hypothesis 2). Additionally, as an exploratory approach it was also planned to investigate other weight-related variables [13, 14].

\section{Materials and methods}

This case-control study was approved by the Ethics Committee of the Medical University before commencement of the study. Written informed consent was obtained from all participants before they were enrolled.

We included patients on the waiting list for hip or knee arthroplasty above 50 years of age. Exclusion criteria for the study and the reference group were as follows: (a) patients with OA or endoprosthesis of the hip for patients in the knee group and vice versa, (b) secondary OA due to hip dysplasia, (c) secondary OA due to trauma, (d) secondary OA due to infection, (e) prolonged corticosteroid therapy, (f) previous chemotherapy, (g) impaired cognitive functions, and (h) language barriers.

The reference group consisted of patients: (a) free of hip/ knee OA in their medical history and (b) free of hip/knee symptoms. We included patients admitted for other reasons (e.g. upper limb surgery) as well as volunteers recruited via information in general practitioners' offices, on the hospital's intranet homepage and in a local newspaper.

All participants underwent a standardized interview to assess their weight at the age of 18 years, their highest and lowest weight in adulthood. It might be speculated whether there would be some recall bias during self-reporting of body weight. However, previous research reported that recall and self-reporting of weight and height are satisfactorily accurate $[15,16]$.

As objective measurements, participants were weighed on a calibrated personal scale, height was measured with a stadiometer and waist circumferences were determined using a measuring tape. Height and weight were measured wearing light indoor clothing without shoes. The body mass index (BMI) was calculated from height and weight values. The waist circumference was measured over the bare skin on the narrowest circumference between the iliac crest and the costal margin; if no such distinction could be made, the circumference at the height of the umbilicus was taken.

Binary logistic regressions were used to determine predictors for OA group membership and the Enter method to calculate the complete model. All models were adjusted for age and sex. 'Maximum-BMI' was used as main indicator and tested for the predictive value. Current BMI was also tested. We evaluated the odds ratios (OR) with their $95 \%$ confidence intervals (CI), the $p$ values and the Nagelkerke $R^{2}\left(R^{2}\right)$. Statistical analyses were performed using the SPSS software, version 18.0. The patients' characteristics were calculated in Excel, Microsoft Office 2003. $p$ values $(p)$ of $<0.05$ were considered significant. In addition to testing our main hypotheses, we used an explorative approach to examine other possible weight-related predictors of OA: we evaluated the effects of weight change (measured as the "maximum-BMI-change" over one's previous adult life (= "maximum-BMI" - "minimum-BMI" after the age of 18 years) and as the "adult-BMI-change" (= "maximumBMI" - "BMI-at-age-18-years") as well as weight distribution indicators, namely, current "waist-circumference" and "waist-circumference-to-height-ratio" (as they have been linked to a metabolic, leptin-associated pathway of OA [17, 18]).

\section{Results}

99 participants were recruited, 49 women and 50 men. 29 patients were in the knee OA group (18 women and 11 men), 30 in the hip OA group (10 women and 20 men), and 40 in the reference group ( 21 women and 19 men) (Table 1). While the mean age in the knee OA group was older [mean 71.4 years, standard deviation (SD) 10.2], than in the reference group (mean 65.1 years, SD 7.3)-which was accounted for by adjusting for age in our binary logistic regression models - the mean age in the hip OA group (mean 65.1 years, SD 7.7), was similar to the reference group.

An increase in 'maximum-BMI', increased the odds ratio for both knee OA (OR 1.2; CI 1.1-1.4; $p=0.005 ; R^{2}=0.36$ ) and hip OA (OR 1.2; CI 1.0-1.3; $p=0.027 ; R^{2}=0.16$ ) (Hypothesis 1).

The mean current BMI of the knee OA group (mean 29.0, SD 4.5) was higher than in the other groups, which was statistically significant in the regression model when compared to the reference group (OR 1.2; CI 1.1-1.4; $p=0.01$; $R^{2}=0.31$ ), but not when compared to the hip OA group (OR $1.1 ; p=0.14)$. The mean current BMI was slightly higher in the hip OA group (mean 27.4, SD 5.5) compared to the reference group (mean 26.1, SD 4.0), but not statistically significant in the binary logistic regression model (OR 1.0; $p=0.312$ ). This means an association of the current BMI to knee OA but not to hip OA (Hypothesis 2).

For our explorative research, we found the following indicators to have a statistically significant predictive power: the only other predictor for hip OA, besides the 'maximum-BMI', was a high lowest BMI over the age of 18 years ('minimum-BMI') (OR 1.3; CI $\left.1.0-1.7 ; p=0.022 ; R^{2}=0.16\right)$. This was also the strongest predictor for knee OA (OR 1.6; CI 1.2-2.2; $p=0.003 ; R^{2}=0.36$ ). With regards to knee $\mathrm{OA}$, all indicators showed a statistically significant predictive value 
Table 1 Patients characteristics

\begin{tabular}{lclcc}
\hline Indicators & Total $(n=99)$ & $\begin{array}{l}\text { Hip OA } \\
\text { Group }(n=30)\end{array}$ & $\begin{array}{l}\text { Knee OA } \\
\text { Group }(n=29)\end{array}$ & $\begin{array}{l}\text { Reference } \\
\text { Group }(n=40)\end{array}$ \\
\hline Age (years) & $66.9(8.8)$ & $65.1(7.7)$ & $71.4(10.2)$ & $65.1(7.3)$ \\
Age BMI min $^{\mathrm{a}}$ & $28.4(14.9)$ & $29.2(17.7)$ & $26.3(11.7)$ & $29.4(14.9)$ \\
Age BMI max $^{\mathrm{b}}$ & $48.3(17.3)$ & $42.8(18.6)$ & $52.3(15.0)$ & $49.4(17.1)$ \\
BMI current $^{\mathrm{c}}$ & $27.3(4.8)$ & $27.4(5.5)$ & $29.0(4.5)$ & $26.1(4.0)$ \\
BMI min $^{\mathrm{d}}$ & $21.7(2.5)$ & $22.3(2.9)$ & $22.4(2.4)$ & $20.7(1.8)$ \\
BMI max $^{\mathrm{e}}$ & $29.5(4.7)$ & $30.1(4.7)$ & $31.3(5.0)$ & $27.7(4.0)$ \\
BMI max change $^{\mathrm{f}}$ & $5.8(6.6)$ & $4.9(7.6)$ & $8.1(5.2)$ & $4.8(6.3)$ \\
BMI max change $\left(1\right.$ year $^{\mathrm{g}}$ & $2.3(2.5)$ & $1.5(2.1)$ & $3.3(3.1)$ & $2.1(2.1)$ \\
BMI change (max-18) & $7.0(4.7)$ & $6.5(5.3)$ & $8.8(4.3)$ & $6.0(4.2)$ \\
Waist circumference $^{\mathrm{h}}$ & $96.7(16.9)$ & $95.7(22.3)$ & $103.8(13.4)$ & $92.3(12.6)$ \\
Waist-to-height ratio & $58.0(8.0)$ & $56.0(12.8)$ & $63.1(7.3)$ & $54.3(7.0)$ \\
\hline
\end{tabular}

Mean and standard deviation (in brackets) are given

$O A$ osteoarthritis

${ }^{a}$ Age BMI min age at BMI minimum

${ }^{\mathrm{b}}$ Age BMI max age at BMI maximum

${ }^{\mathrm{c}} \mathrm{BMI}$ current $=$ weight $($ kilogram, measured $) /$ height $^{2}$ (meter, measured)

${ }^{\mathrm{d}} \mathrm{BMI} \min =$ "minimum-BMI" = lowest reported weight/height ${ }^{2}$ (according to the questionnaire)

eBMI max = "maximum BMI" = highest reported weight/height ${ }^{2}$

${ }^{\mathrm{f}}$ BMI max change = "maximum BMI change" = BMI max - BMI min

${ }^{\mathrm{g}}$ BMI max change $(1$ year) = "maximum BMI change within 1 year" = reported max weight in 1 year/ height $^{2}$

${ }^{\text {h}}$ BMI change $(\max -18)=$ "adult-BMI-change" = BMI maximum at age of 18 years (reported)

Table 2 Comparison of predictive value if indicators and obesity among hip, knee and reference group

\begin{tabular}{|c|c|c|c|c|c|c|c|c|c|c|c|c|}
\hline \multirow[t]{2}{*}{ Indicators } & \multicolumn{4}{|c|}{ HIP OA to reference group } & \multicolumn{4}{|c|}{ Knee OA to reference group } & \multicolumn{4}{|c|}{ Knee OA to hip OA group } \\
\hline & $p$ value & OR & $95 \% \mathrm{CI}$ & $R^{2}$ & $p$ value & OR & $95 \% \mathrm{CI}$ & $R^{2}$ & $p$ value & OR & $95 \% \mathrm{CI}$ & $R^{2}$ \\
\hline BMI max & 0.027 & 1.154 & $1.017-1.310$ & 0.16 & 0.005 & 1.220 & $1.063-1.400$ & 0.359 & 0.241 & 1.081 & $0.949-1.231$ & 0.275 \\
\hline BMI max change 1 year & 0.329 & 0.880 & $0.682-1.137$ & 0.067 & 0.116 & 1.187 & $0.959-1.471$ & 0.234 & 0.011 & 1.447 & $1.086-1.926$ & 0.396 \\
\hline Current BMI & 0.312 & 1.056 & $0.950-1.172$ & 0.068 & 0.010 & 1.201 & $1.045-1380$ & 0.313 & 0.138 & 1.104 & $0.969-1.258$ & 0.292 \\
\hline BMI change (max-18) & 0.392 & 1.043 & $0.947-1.150$ & 0.063 & 0.011 & 1.186 & $1.040-1.352$ & 0.317 & 0.061 & 1.129 & $0.994-1.282$ & 0.317 \\
\hline BMI max change & 0.178 & 1.086 & $0.963-1.224$ & 0.086 & 0.041 & 1.165 & $1.007-1.349$ & 0.272 & 0.306 & 1.077 & $0.935-1.240$ & 0.267 \\
\hline BMI min. & 0.022 & 1.313 & $1.040-1.657$ & 0.155 & 0.003 & 1.583 & $1.164-2.153$ & 0.36 & 0.552 & 1.069 & $0.857-1.334$ & 0.254 \\
\hline Waist circumference & 0.850 & 1.003 & $0.972-1.036$ & 0.05 & 0.002 & 1.091 & $1.032-1.153$ & 0.382 & 0.038 & 1.056 & $1.003-1.111$ & 0.364 \\
\hline Waist-to-height ratio ${ }^{a}$ & 0.682 & 1.012 & $0.958-1.069$ & 0.052 & 0.001 & 1.184 & $1.073-1.307$ & 0.437 & 0.032 & 1.105 & $1.009-1.211$ & 0.376 \\
\hline
\end{tabular}

OR odds ratio, $95 \% \mathrm{Cl} 95 \%$ confidence interval, $R^{2}$ Nagel Kerkes $R^{2}$

${ }^{a}$ Waist-to-height ratio $=$ waist circumference/height

(Table 2). When comparing the knee OA to the hip OA group directly, the waist distribution indicators showed a significant association with knee OA: the "waist-toheight-ratio' (OR 1.1; CI 1.0-1.2; $p=0.032 ; R^{2}=0.38$ ) showed a similar predictive value as the "waist-circumference' alone (OR 1.1; CI 1.0-1.1; $p=0.038 ; R^{2}=0.36$ ).

\section{Discussion}

The most important findings of the study were a significant association between OA of both knee and hip with the 'maximum-BMI' over one's lifetime. It is also regarded as important finding that the 'current BMI', was associated 
only with knee OA but not with hip OA. Moreover, the predictive value (OR) of the 'maximum-BMI' was greater than that of the 'current BMI' in knee OA. The findings are interpreted in a way that the maximum BMI in one's lifetime is a more appropriate predictor than the current BMI to assess the risk of hip and knee OA.

With regard to the parameter 'maximum BMI' over one's lifetime, we tried to compare our findings with what was previously published. However, to our best knowledge, no previous investigators dealt with the relationship between 'maximum BMI' and OA. As the 'maximum-BMI' explains only $36 \%$ of the variance in our knee OA to reference group model and $16 \%$ of the variance in our hip OA to reference group model, further factors need to be investigated and included in a future risk assessment tool like a risk calculator.

'Current BMI' was only associated with OA of the knee in our study population. This supports previous research from Reijman et al. and Grotle et al. [11, 12] and contradicts findings from Wang et al. and Lohmander et al. [9, 10]. Obesity was reported as important risk factor for the onset of OA of the knee joint [19-21] and a questionable risk factor for the development of OA of the hip joint [12, 22]. Various studies have demonstrated that the current BMI was related to knee $\mathrm{OA}$ and that weight loss improves symptoms and functional capacity [12, 23-25]. Biomechanical studies tried to investigate why the knee joint in overweight persons was more affected by OA than the hip joint. A study by Amiri et al. found, that obesity caused a prolonged activation of quadriceps and gastrocnemii, which can result in a prolonged knee joint contact loading, and thereby may contribute to the predisposition of the knee joint to development and progression of OA [26]. This finding is supported by a study by Pamukoff et al. [27]. They found that obesity may contribute to knee OA because of a greater vertical loading in gait biomechanics [27]. Current BMI was found to be a questionable risk factor for the onset of OA of the hip joint $[12,22]$. Overall, in the current literature is consensus, that weight loss is protective against the development of degenerative musculoskeletal conditions [19, 23-25].

In the additional, explorative data analysis, we also found both weight distribution indicators ("waist-circumference" and "waist-to-height-ratio") to be significant predictors of group membership for the knee OA versus the hip OA group. This data possibly indicates that the knee is more vulnerable to metabolic damage to joint and cartilage, which is associated with a higher intra-abdominal fat mass [28]. Like previous studies [18, 29, 30], we also found an association between these weight distribution indicators with knee OA compared to the reference group. Additionally, we found that the knee seems to be significantly more vulnerable to the amount of intra-abdominal fat than the hip, possibly indicating a stronger leptin-associated pathogenesis of knee OA.
'Waist-circumference-to-height-ratio' and 'waist-circumference' may help distinguish whether there is a higher risk of getting knee OA than hip OA. Further investigation of these indicators is needed, to confirm and clarify the interpretation of the findings of our exploratory data analyses.

As limitations of our study, a potential selection bias in our reference group has to be acknowledged. It is possible that more health conscious people volunteered in that group. Another limitation is the study design (case-control study) with typical limitation in comparison with prospective studies. Another limitation is that we neglected the effect of leg malalignment in our model. Moreover, current BMI, waist-circumference, and waist-circumference-to-heightratio measured at study entry could have been biased by limited physical activity associated with OA. It might also be speculated whether there would be some recall bias during self-reporting of body weight. However, previous research reported that recall and self-reporting of weight and height are satisfactorily accurate $[15,16]$.

Based on our findings, it is concluded that the maximum BMI over one's lifespan is a better predictor of OA of the hip or the knee than the current BMI. These findings support the use of the most sustainable methods of weight control. The knee joint seems to be more sensitive to obesity as current BMI was associated only with knee OA but not with hip OA.

Acknowledgements Open access funding provided by University of Innsbruck and Medical University of Innsbruck. This research did not receive any specific grant from funding agencies in the public, commercial, or not-for-profit sectors.

\section{Compliance with ethical standards}

Conflict of interest No benefits in any form have been received or will be received from a commercial party related directly or indirectly to the subject of this article.

Funding The authors did not receive any outside funding or grants in support of their research for or preparation of this work. Neither they nor a member of their immediate families received payments or other benefits or a commitment or agreement to provide such benefits from a commercial entity. No commercial entity paid or directed, or agreed to pay or direct, any benefits to any research fund, foundation, division, center clinical practice, or other charitable or nonprofit organization with which the authors, or a member of their immediate families, are affiliated or associated.

Open Access This article is distributed under the terms of the Creative Commons Attribution 4.0 International License (http://creativecommons.org/licenses/by/4.0/), which permits unrestricted use, distribution, and reproduction in any medium, provided you give appropriate credit to the original author(s) and the source, provide a link to the Creative Commons license, and indicate if changes were made. 


\section{References}

1. Felson DT, Lawrence RC, Hochberg MC, McAlindon T, Dieppe PA, Minor MA, Blair SN, Berman BM, Fries JF, Weinberger M, Lorig KR, Jacobs JJ, Goldberg V (2000) Osteoarthritis: new insights. Part 2: treatment approaches. Ann Intern Med 133(9):726-737

2. Global Burden of Osteoarthritis in the year 2000 (2002) WHO. http://www.who.int/healthinfo/statistics/bod_osteoarthritis.pdf

3. Kotlarz H, Gunnarsson CL, Fang H, Rizzo JA (2009) Insurer and out-of-pocket costs of osteoarthritis in the US: evidence from national survey data. Arthritis Rheum 60(12):3546-3553

4. van Baal PH, Polder JJ, de Wit GA, Hoogenveen RT, Feenstra TL, Boshuizen HC, Engelfriet PM, Brouwer WB (2008) Lifetime medical costs of obesity: prevention no cure for increasing health expenditure. PLoS Med 5(2):e29

5. Sowers MF, Yosef M, Jamadar D, Jacobson J, Karvonen-Gutierrez C, Jaffe M (2008) BMI vs. body composition and radiographically defined osteoarthritis of the knee in women: a 4-year follow-up study. Osteoarthr Cartil OARS Osteoarthr Res Soc 16(3):367-372

6. Abbate LM, Stevens J, Schwartz TA, Renner JB, Helmick CG, Jordan JM (2006) Anthropometric measures, body composition, body fat distribution, and knee osteoarthritis in women. Obesity 14(7):1274-1281 ((Silver Spring, Md))

7. Felson DT (1995) Weight and osteoarthritis. J Rheumatol 43:7-9

8. Felson DT (1996) Weight and osteoarthritis. Am J Clin Nutr 63(3 Suppl):430S-432S

9. Wang Y, Simpson JA, Wluka AE, Teichtahl AJ, English DR, Giles GG, Graves S, Cicuttini FM (2009) Relationship between body adiposity measures and risk of primary knee and hip replacement for osteoarthritis: a prospective cohort study. Arthritis Res Ther 11(2):R31

10. Lohmander LS, Gerhardsson de Verdier M, Rollof J, Nilsson PM, Engstrom G (2009) Incidence of severe knee and hip osteoarthritis in relation to different measures of body mass: a population-based prospective cohort study. Ann Rheum Dis 68(4):490-496

11. Grotle M, Hagen KB, Natvig B, Dahl FA, Kvien TK (2008) Obesity and osteoarthritis in knee, hip and/or hand: an epidemiological study in the general population with 10 years follow-up. BMC Musculoskelet Disord 9:132

12. Reijman M, Pols HA, Bergink AP, Hazes JM, Belo JN, Lievense AM, Bierma-Zeinstra SM (2007) Body mass index associated with onset and progression of osteoarthritis of the knee but not of the hip: the Rotterdam Study. Ann Rheum Dis 66(2):158-162. doi:10.1136/ard.2006.053538

13. Gandhi R, Dhotar H, Tsvetkov D, Mahomed NN (2010) The relation between body mass index and waist-hip ratio in knee osteoarthritis. Can J Surg 53(3):151-154

14. Liu B, Balkwill A, Banks E, Cooper C, Green J, Beral V (2007) Relationship of height, weight and body mass index to the risk of hip and knee replacements in middle-aged women. Rheumatology 46(5):861-867 (Oxford, England)

15. Dekkers JC, van Wier MF, Hendriksen IJ, Twisk JW, van Mechelen W (2008) Accuracy of self-reported body weight, height and waist circumference in a Dutch overweight working population. BMC Med Res Methodol 8:69

16. Tehranifar P, Liao Y, Flom JD, Terry MB (2009) Validity of selfreported birth weight by adult women: sociodemographic influences and implications for life-course studies. Am J Epidemiol 170(7):910-917
17. Stannus OP, Jones G, Quinn SJ, Cicuttini FM, Dore D, Ding C (2010) The association between leptin, interleukin-6, and hip radiographic osteoarthritis in older people: a cross-sectional study. Arthritis Res Ther 12(3):R95

18. Gandhi R, Takahashi M, Syed K, Davey JR, Mahomed NN (2010) Relationship between body habitus and joint leptin levels in a knee osteoarthritis population. J Orthop Res 28(3):329-333

19. Zhou ZY, Liu YK, Chen HL, Liu F (2014) Body mass index and knee osteoarthritis risk: a dose-response meta-analysis. Obesity (Silver Spring) 22(10):2180-2185. doi:10.1002/oby.20835

20. Zheng H, Chen $\mathrm{C}$ (2015) Body mass index and risk of knee osteoarthritis: systematic review and meta-analysis of prospective studies. BMJ Open 5(12):e007568. doi:10.1136/ bmjopen-2014-007568

21. Lee S, Kim TN, Kim SH, Kim YG, Lee CK, Moon HB, Koh EM, Yoo B (2015) Obesity, metabolic abnormality, and knee osteoarthritis: a cross-sectional study in Korean women. Mod Rheumatol 25(2):292-297. doi:10.3109/14397595.2014.939393

22. Reyes C, Leyland KM, Peat G, Cooper C, Arden NK, PrietoAlhambra D (2016) Association between overweight and obesity and risk of clinically diagnosed knee, hip, and hand osteoarthritis: a population-based cohort study. Arthritis Rheumatol 68(8):18691875. doi:10.1002/art.39707

23. Bliddal M, Pottegard A, Kirkegaard H, Olsen J, Jorgensen JS, Sorensen TI, Dreyer L, Nohr EA (2016) Association of pre-pregnancy body mass index, pregnancy-related weight changes, and parity with the risk of developing degenerative musculoskeletal conditions. Arthritis Rheumatol 68(5):1156-1164. doi:10.1002/ art.39565

24. Runhaar J, van Middelkoop M, Reijman M, Willemsen S, Oei EH, Vroegindeweij D, van Osch G, Koes B, Bierma-Zeinstra SM (2015) Prevention of knee osteoarthritis in overweight females: the first preventive randomized controlled trial in osteoarthritis. Am J Med 128(8):888-895 e884. doi:10.1016/j.amjmed.2015.03.006

25. Isla Pera P, Ferrer MC, Nunez Juarez M, Nunez Juarez E, Macia Soler L, Lopez Matheu C, Rigol Cuadra A, Perez MH, Marre D (2016) Obesity, knee osteoarthritis, and polypathology: factors favoring weight loss in older people. Patient Prefer Adherence 10:957-965. doi:10.2147/PPA.S92183

26. Amiri P, Hubley-Kozey CL, Landry SC, Stanish WD, Astephen Wilson JL (2015) Obesity is associated with prolonged activity of the quadriceps and gastrocnemii during gait. J Electromyogr Kinesiol 25(6):951-958. doi:10.1016/j.jelekin.2015.10.007

27. Pamukoff DN, Lewek MD, Blackburn JT (2016) Greater vertical loading rate in obese compared to normal weight young adults. Clin Biomech (Bristol, Avon) 33:61-65. doi:10.1016/j. clinbiomech.2016.02.007

28. Monti V, Carlson JJ, Hunt SC, Adams TD (2006) Relationship of ghrelin and leptin hormones with body mass index and waist circumference in a random sample of adults. J Am Diet Assoc 106(6):822-828 (quiz 829-830)

29. Gandhi R, Dhotar H, Tsvetkov D, Mahomed NN (2010) The relation between body mass index and waist-hip ratio in knee osteoarthritis. Can J Surg 53(3):151-154

30. Han TS, McNeill G, Seidell JC, Lean ME (1997) Predicting intraabdominal fatness from anthropometric measures: the influence of stature. Int J Obes Relat Metab Disord 21(7):587-593 